\title{
STUDY AND REALIZATION OF IMAGE SEGMENTATION ON THE COTTON FOREIGN FIBERS
}

\author{
Wenxiu Zheng, Jinxing Wang ${ }^{*}$, Shuangxi Liu, Xinhua Wei \\ Mechanical and Electronic Engineering College, Shandong Agricultural University, Taian, \\ Shandong Province, P.R. China 271018 \\ * Corresponding author, Address: Mechanical and Electronic Engineering College, Shandong \\ Agricultural University, Taian 271018, Shandon Province, P.R.China, Tel: +86-538- \\ 8246826, Fax: +86-538-8246107,Email:jinxingw@163.com
}

Abstract: A method of foreign fibers image segmentation based on Mean shift dilation and filtering algorithm is presented. For the representative gray images of hair shicken feather and mixed foreign fibers, the Mean shift algorithm is used to carry on image segmentation; then dilation and filtering process is carried on to the divided image element. In this way the precise image segmentation of foreign fibers is realized. It's proved by experiments that the image segmentation method proposed by this article can suppress the noise well, and the segmentation results are satisfied for all kinds of foreign fibers image.

Keywords: Foreign fibers, Histogram analysis, Image segmentation, Mean shift

\section{INTRODUCTION}

Foreign fibers refer to the non-cotton fibers and the colored fibers which are mixed in the process of raw cotton production v processing and circulation, such as chemical fiber、hair、silk、 linen、plastic film、plastic rope、dyeing lines and so on( Li Bidan et al.,2006). Although the net content is low, there is a big impact on the cotton industries. Now the general selection of manual work has low efficiency and consumes a lot of time. The professional investigation estimates that a medium cotton enterprise uses

Please use the following format when citing this chapter:

Zheng, W., Wang, J., Liu, S. and Wei, X., 2009, in IFIP International Federation for Information Processing, Volume 294, Computer and Computing Technologies in Agriculture II, Volume 2, eds. D. Li, Z. Chunjiang, (Boston: Springer), pp. 1067-1075. 
300-400 people to select the foreign fibers everyday, and the annual cost of this item amounts to more than 200 million Yuan. Whatsoever the selection of foreign fibers is like to look for a needle in a sea which results in the eye weariness of the workers for glaring at the white cotton for such a long time and finally the foreign fibers which are not sorted still cause the problem of product quality(Kang et al.,2002; Wang Xinlong et al.,2002).So applying the machine vision technology to research rapid recognition of foreign fibers are of great significance, for it can improve the quality of cotton and promote the development of cotton textile industries. The foreign fibers detection system based on machine vision is the key technology, thus this paper mainly research the image segmentation technology of foreign fibers (Jiao Wenxing et al., 2003).

The aim of image segmentation technology is to distinguish the different special meaning areas as these areas are not intersect mutually,

image segmentation is not only one of the great important contents in the area of image processing, but also a classic problem in machine vision. For image analysis and understanding, the original image is transformed a more abstract form through separate objectives 、 extract parameters and survey

parameters. The common methods of image segmentation have threshold value segmentation 、 region growing region splitting and merging, edge detection and boundary tracking and so on. Because of the complexity of the imaging and the information included in the image, though the researchers proposed many segmentation methods, so far there is not a general segmentation method (Zhu Zhigang et al., 2003; Zhang Xiaolu et al., 2005).

In this paper, a new method of foreign fibers image segmentation based on mean shift dilation and filtering algorithm is presented, it can suppress the noise well. Through the histogram analysis to the foreign fibers image, the gray scale distributed situation was determined and this image segmentation method was used to realize the image segmentation. The results indicate that this method has the characteristics of high speed and accuracy (Chen Donglan et al., 2003).

\section{EXPERIMENTAL MATERIALS}

This experiment chooses 14 kinds of foreign fibers, such as the chicken feather 、 the white paper 、 the fluorescent polypropylene silk 、 nonfluorescent green-grey polypropylene silk 、 non-fluorescent white polypropylene silk , the white chemical fiber silk , the colored chemical fiber cloth strip 、 the colored cotton cloth strip 、 the hemp rope the hair the candy wrapper、the colored sheep yarn 、 the white plastic bag 、 mulching plastic and so on, and full amount of ginned cotton which doesn't 

Fibers

contain foreign fibers. After fully-smashing by the ginned cotton machine, the uninterrupted uniform cotton layer of 80 centimeters wide and two millimeters thick were formed. In the process of smashing the above foreign fiber samples were put in gradually, also the layer of foreign fibers were preserved after the process, then the scanner would be applied to carry on the image scanning on these layers of cotton samples. As a result, 40 foreign fiber images of 24 true colors whose size is $1700 \times 2344$ were produced.

\section{HISTOGRAM ANALYSIS}

In order to extract the foreign fibers smoothly, the 40 samples were taken to make the histogram analysis. As the image samples are the 24 true color images, then before the gray histogram analysis, the image type was necessary to convert, 24 true color images will be converted to 256 gray images (Kapur et al., 1985). So the gray images of hair 、 chicken feather and mixed foreign fibers were acquired and their histogram analysis was done. The following Fig.1-3 are the gray images and histograms:
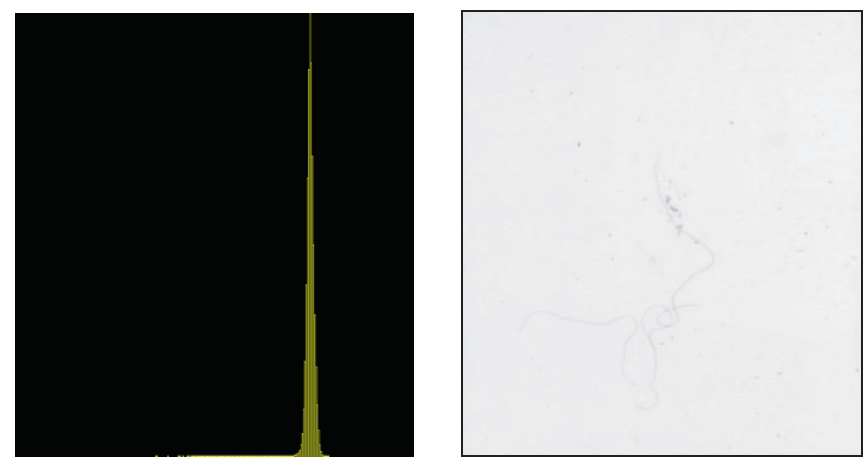

Fig. 1 hair's gray image and histogram
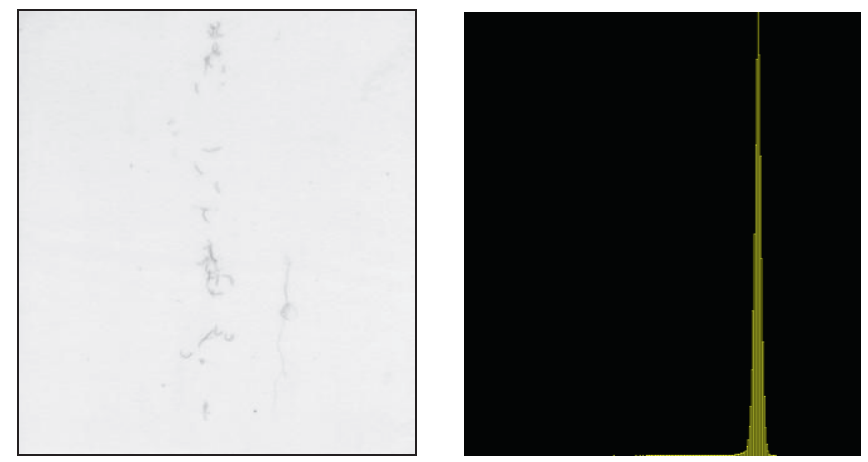

Fig.2 chicken feather's gray image and histogram 

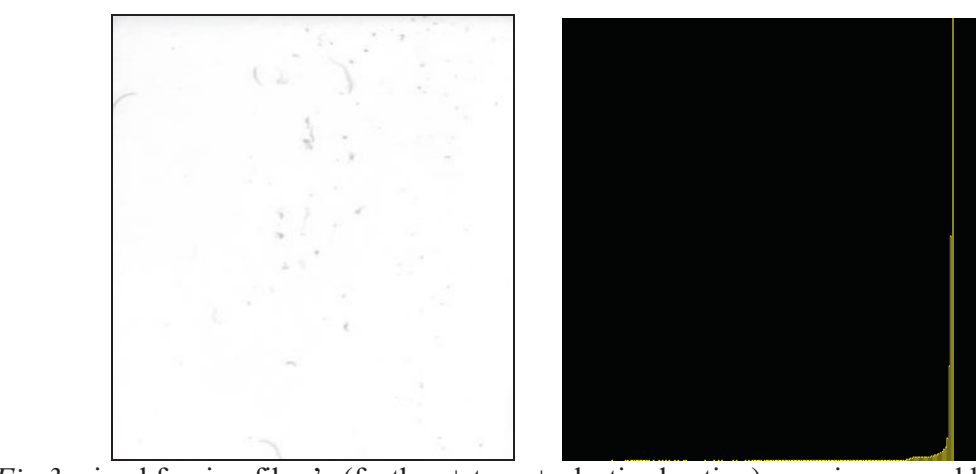

Fig. 3 mixed foreign fiber's (feather + tape + plastic sheeting) gray image and histogram

Based on the research of gray histograms from Fig.1 to Fig.3, the following conclusions are drawn:

(1) All histograms present a single peak distribution, and the aggregation is very high. These show that the gray values of cotton and foreign fibers mix together, and the cotton's background occupies the overwhelming superiority in the whole image, however the foreign fibers' has very small proportion in the whole image;

(2) The gray values of cotton background are between $230 \sim 255$ in general and the gray values of foreign fibers are less than or equal to 230 .

\section{THE RESEARCH OF FOREIGN FIBER'S EXTRACTION METHOD}

From the results of histogram analysis, the foreign fibers can't be extracted by using the traditional single-threshold value segmentation or multi-threshold value segmentation, for the single-threshold value segmentation needs the histograms to show one kind of twin peaks or the multi-peak distribution, however the multi-threshold value segmentation needs to split the image into many sub-images and in order to discriminate the objects, each sub-image was needed to be marked, what's more, after threshold value segmentation, the adjacent boundary of sub-images may have the grayscale discontinuity(Chen Donglan et al.,2003). Therefore the method of Mean-shift adaptive threshold value segmentation was decided to be chosen to complete the segmentation of the foreign fibers.

Mean shift process is a method of kernel density estimation, which is based on the Parzen window method to examine the probability density function in the pattern recognition, corresponding in the window function $\varphi(x)$ of the Parzen window method, and the definition kernel function $\mathrm{K}(\mathrm{x})$.In most cases what we care about is the symmetry of kernel function, which could be expressed for the following forms: 
Fibers

$$
K(x)=C_{k, d} k\left(\|x\|^{2}\right) \text {. And } C_{k, d} \text { is constant. }
$$

Here take the Gauss kernel function as an example:

$$
K(x)=(2 \pi)^{-d / 2} \exp \left(-\frac{1}{2}\|x\|^{2}\right)
$$

Obtain the convergent recurrence formula and the Mean shift vector:

$$
\begin{gathered}
y_{i+1}=\frac{\sum_{i=1}^{n} x_{i} g\left(\left\|\frac{x-x i}{h}\right\|^{2}\right)}{\sum_{i=1}^{n} g\left(\left\|\frac{x-x i}{h}\right\|^{2}\right)} \\
\mathrm{m}_{h, G}=\frac{\sum_{i=1}^{n} x_{i} g\left(\left\|\frac{x-x i}{h}\right\|^{2}\right)}{\sum_{i=1}^{n} g\left(\left\|\frac{x-x i}{h}\right\|^{2}\right)}-x=y_{i+1}-y_{i}
\end{gathered}
$$

The Mean shift method has many strong points. The path of the Mean shift vector movement towards convergence point is a smooth track and the angle of two consecutive vectors is usually less than 90 degrees. This advantage guarantees the stability of the convergence, and compared with other fast rise ways, the gentle path of the Mean shift can complete the image segmentation rapidly; achieving the purpose of rapid and natural portray the real lines of nature objects. Based on the above characteristics, the application of the Mean shift technology to the foreign fibers image segmentation can divide the target sector exactly and obtain the accurate foreign fibers image (Jensen et al., 2002; Jason et al., 2008).

In the course of the research, regarding the dispersion of the image information, finally 13 pixels Mean-shift adaptive threshold segmentation method was selected to carry on image segmentation through repeated experiments (Zhu Zhigang et al., 2003).The following Fig.4-6 are the processing effect: 


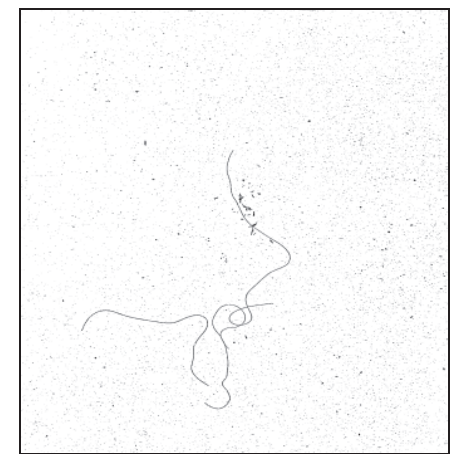

Fig.4 Mean shift effect chart of hair gray image

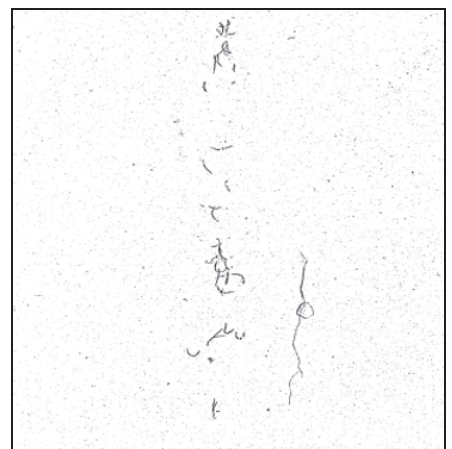

Fig. 5 Mean shift effect chart of chicken feather gray image

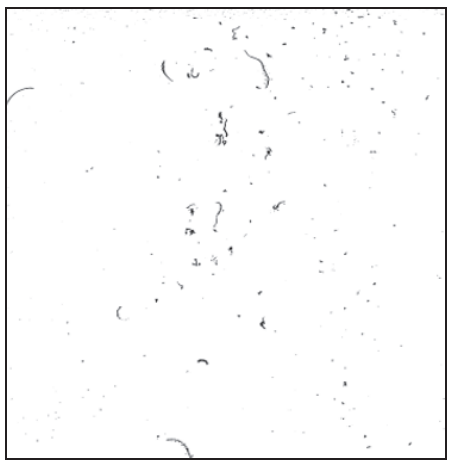

Fig. 6 Mean shift effect chart of mixed foreign fiber (feather + tape + plastic sheeting) gray image

\section{THE ENHANCEMENT PROCESSING OF FOREIGN FIBERS' IMAGE}

Contrasting the original gray images, results could be found:

(1) Salt and pepper noise exists in the local of light uneven;

(2) There is a phenomenon of excessive processing.

In order to obtain the image which is closer to the original gray image, it is necessary to unify some processing methods to optimize or enhance the image:

(1) Median filtering technology was used to weaken the salt and pepper noise;

(2) The phenomenon of excessive processing is due to the band width of the Mean-shift algorithm is fixed and the change of two images' background.

Based on the above two points, dilation process was carried on to the image element first, so the part phenomenon of excessive processing was revised and the region of foreign fibers was made to connect as far as 
Fibers

possible; then filtering process was used. After repeated testing, $3 \times 3$ rectangular structural element was decided to use to deal with a dilation process; then two $7 \times 7$ median filtering processes were used again, the experiments show that this approach can get more satisfactory results (Zhu Zhigang et al., 2003; Ying Xu et al., 1998). The effect of after dilation and filtering process is shown in Fig.7-9.
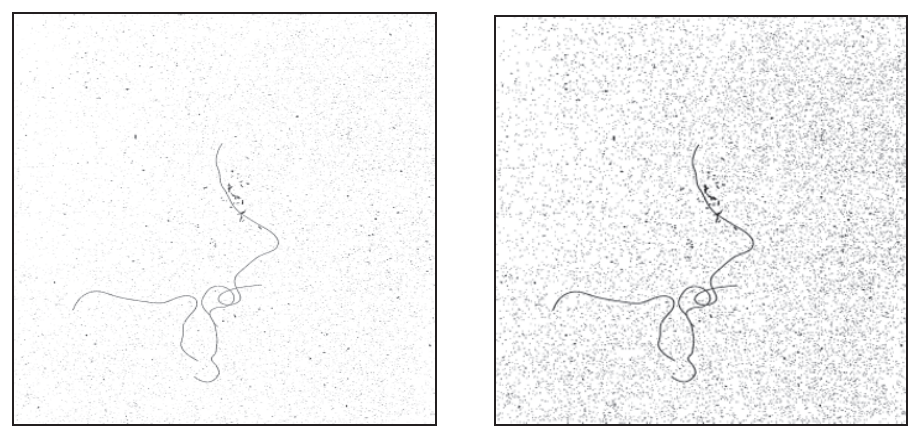

Fig. 7 the dilation and filtering effect chart of hair Mean-shift image
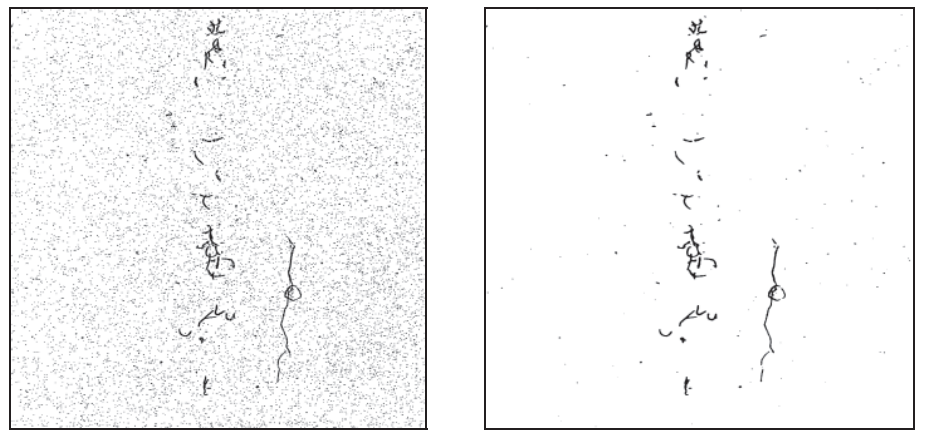

Fig. 8 the dilation and filtering effect chart of chicken feather Mean-shift image
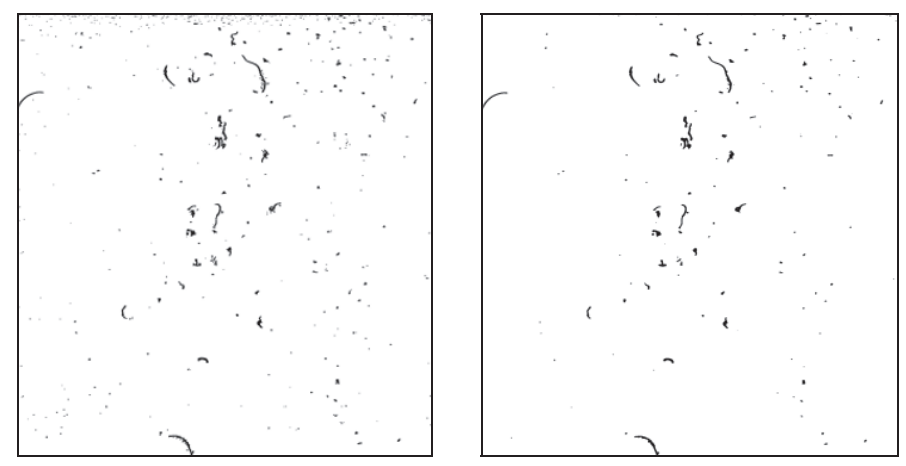

Fig. 9 the dilation and filtering effect chart of mixed foreign fibers (feather + tape + plastic sheeting) Mean-shift image 
The precise extraction of foreign fibers was completed after the dilation and filtering process on the foreign fibers Mean-shift image. Contrasting the original image with the final processing results, a discovery could be made:

(1) This method effectively extracts the foreign fibers;

(2) This method depends less on the environment illumination;

(3) Although effective, there are still a small portion of non foreign fibers are extracted, which could be considered to join the revision in the following work.

Through the above processes, the extraction of foreign fibers is precisely completed by using the method of foreign fibers image segmentation based on Mean shift dilation and filtering algorithm, the results indicate that this method has the characteristics of high speed and accuracy.

\section{CONCLUSION}

(1) Contrasting the final effect chart with the original image, it could be concluded that the extraction of foreign fibers was effectively completed by Mean-shift adaptive threshold segmentation method and the enhancement processing of foreign fibers image was completed by the dilation and filtering process. This series of processing methods extract the foreign fibers effectively;

(2) The image segmentation method which this paper presents is an important addition to the existing methods, and it can obtain the better segmentation effect for different types of image, compared with other segmentation methods.

\section{ACKNOWLEDGEMENTS}

The project supported by the National Science and Technology Pillar Program in the Eleventh Five-year Plan Period.(2006BAD11A14-3)

\section{REFERENCES}

Chen Donglan, Liu Jingnan, Yu Ling-ling. Comparison of image segmentation threshold method[J].Machine Building \& Automation, 2003, 1(1):77 80

Jason E. Fritts, Hui Zhang, et al. Image segmentation evaluation: A survey of unsupervised methods. Computer Vision and Image Understanding, 2008, 110 (2) :260 280

Jensen K L, Carstensen J M. Fuzz and pills evaluated on knitted textiles by image analysis [J].Textile Res J, 2002, 72(1): 34 38

Jiao Wenxing, Pan Tianli, Li Yue. Application of computer vision technique in agricultural products quality inspection. Shanxi Journal of Agricultural Sciences, 2003, (5):29 33 
Fibers

Kang T J, Kim S C. Objection evaluation of the trash and color of raw cotton by image processing and neural network[J].Textile Res J, 2002, 72(9): 776 782

Kapur J N, Sahoo P K. A new method for gray-level picture threshold using the entropy of the histogram[J].Computer Vision, Graphics, and Image Processing, 1985, 29(3):273 285

Li Bidan, Ding Tianhuai, Jia Dongyao. Design of a Sophisticated Foreign Fiber Separator [J]. Agricultural Machinery Journal, 2006, 37 (1) : 107-110

Wang Xinlong, Li Na. Summary of the foreign fibers [J]. China Cotton Processing, 2002, (5) :29 30

Ying $\mathrm{Xu}$, Victor Olman, et al. A segmentation algorithm for noisy images: design and evaluation. Pattern Recognition Letters, 1998, 19 (13):1213 1224

Zhang Xiaolu, Han Liqun. Application of Computer Vision Technology to Fiber Identification [J]. Journal of Beijing Technology and Business University, 2005, 23 (2) :43-45

Zhu Zhigang, Shi Dingji, et al. Digital Image Processing[M].Publishing House of Electronics Industry, 2003 